\title{
Pulsed Vector Magnetic Potential Field Existence
}

\author{
Ivan Rampl ${ }^{1}$, Lukáš Palko ${ }^{1}$, Pavel Hyršl ${ }^{2}$, Libor Vojtek ${ }^{2}$ \\ ${ }^{1}$ ENJOY Ltd., Brno, Czech Republic; ${ }^{2}$ Masaryk University, Brno, Czech Republic. \\ Email: ramp1@enjoy.cz,palko@enjoy.cz,hyrs1@sci.muni.cz, libor.vojtek@email.cz
}

Received April 23 $3^{\text {rd }}, 2012$; revised May $27^{\text {th }}, 2012$; accepted June $11^{\text {th }}, 2012$

\begin{abstract}
Experimental confirmation discussed the effect of the immediate surroundings of a pulse-powered toroidal coil on biological material which was placed in an environment without the influence of electromagnetic force.
\end{abstract}

Keywords: Vector Magnetic Potential; Toroidal Coil; Cell Viability; Cell Metabolism; Bacterium; Escherichia coli

\section{Introduction}

The vector magnetic potential $\mathbf{A}(\mathrm{Wb} / \mathrm{m})$ is a three-dimensional vector field whose curl is the magnetic field (B-field)

$$
\mathbf{B}=\operatorname{rot} \mathbf{A}=\nabla \times \mathbf{A} \quad\left(\mathrm{Wb} / \mathrm{m}^{2}\right) .
$$

Together with the electric potential $\phi$, the vector magnetic potential A can also be used to specify the electric field (E-field) when electric charges are in motion (i.e. electric currents are present)

$$
\mathbf{E}=-\nabla \phi-(\delta \mathbf{A} / \mathrm{dt}) \quad(\mathrm{V} / \mathrm{m}) .
$$

Defined in this way, $\mathbf{B}$ can be obtained from "Equation (1)", but there are an infinite number of vector $\mathbf{A}$ fields that satisfy "Equation (1)" so the definition of this vector field requires an additional specification.

The vector magnetic potential A can exist apart and isolated in space from the B-field which gives rise to its existence. As a direct result of this peculiar attribute, the vector magnetic potential has been assumed to be the fundamental action mechanism for the gravitational force.

Professor Albert Shadowitz (1988) states, among other things, that "A provides tremendous advantages when variations with time are considered" [1]. This statement gave rise to an experiment that would prove the force effect of Pulsed Vector Magnetic Potential (PVMP) on living cells. If such an effect on cell metabolism existed at all, the viability of living cells should change due to this effect in comparison with the viability of identical but unaffected cells, on the assumption that the effect of electromagnetic force in the same area would be prevented [2]. The source of PVMP-field is a toroidal coil supplied with periodic pulsed signal, with the B-field concentrated only in the ferromagnetic core while the E-field occurs in the surroundings of the coil.

\section{Materials and Methods}

\subsection{General Description of the Technological Part of Experiment}

For the intended experiment, a device was constructed with a large applicator consisting of two toroidal coils, each of which has an outer dia. of ca $600 \mathrm{~mm}$, the core of transformer steel $\left(\mathrm{B}_{\text {rem }}=1.442 \mathrm{~T}, \mathrm{H}_{\text {coer }}=13.4 \mathrm{~A} / \mathrm{m}\right.$, core losses $\mathrm{W}_{\text {hyst }}=121.7 \mathrm{~J} / \mathrm{m}^{3}$ ) and winding along the whole circumference. The reasons for building such large coils were 1) the relatively high value of E-field intensity that can be obtained (according to the computer modelling performed); 2) the large probe with measuring instrument sensors for the verification of the E-field intensity and distribution values obtained by simulation; 3 ) the sufficiently large area for locating a large 96-wells microtitration plate with removable wells for holding bacterial suspension.

Electrical parameters of the applicator and the generator:

1) Each of the two toroidal coils is formed by 20 turns with the inductance $\mathrm{L}_{20}=1.2 \mathrm{mH}$ and resistance $\mathrm{R}_{20}=12$ $\mathrm{m} \Omega$;

2) The pulsed power generator generates rectangular current pulses $\mathrm{I}_{\text {pulse }}=(0-10) \mathrm{A}$, pulse width $\mathrm{T}_{\text {pulse }}=(1-$ $10) \mathrm{ms}$, and with the possibility of setting the mark-tospace ratio to $1: 8,1: 16,1: 32$, and 1:64.

\subsubsection{Calculation Simulation}

To give an idea of the distribution of E-field and PVMPfield, the following Figures 1(c) and (d) show the case of a pair of toroidal coils of 20 turns each, connected in series and supplied with square current pulses with the width $\mathrm{T}_{\text {pulse }}=1 \mathrm{~ms}$, mark-to-space ratio $1: 32$, current pulse size $\mathrm{I}_{\text {pulse }}=10 \mathrm{~A}$ as shown in Figure 2, and identical magnetic flux direction. The experiment de- 


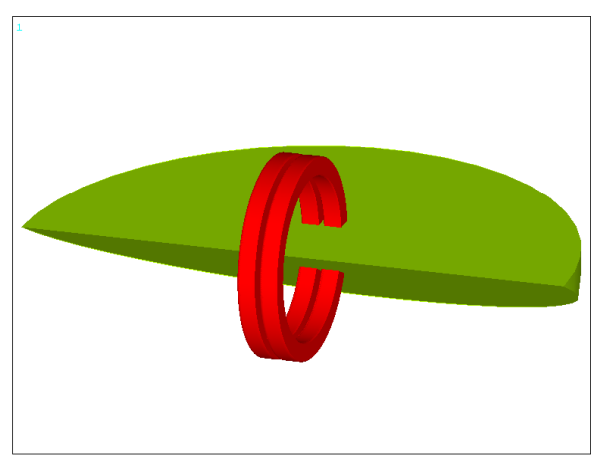

(a)

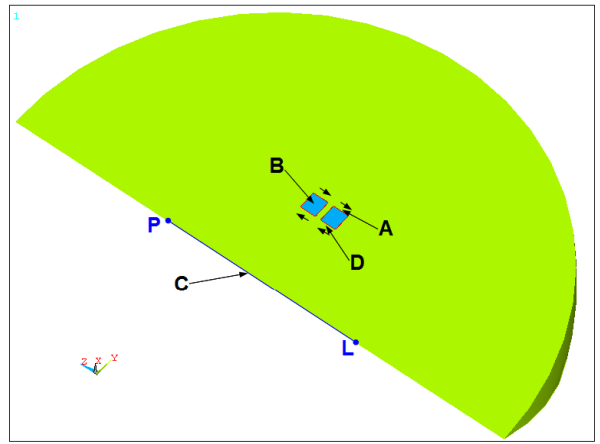

(b)

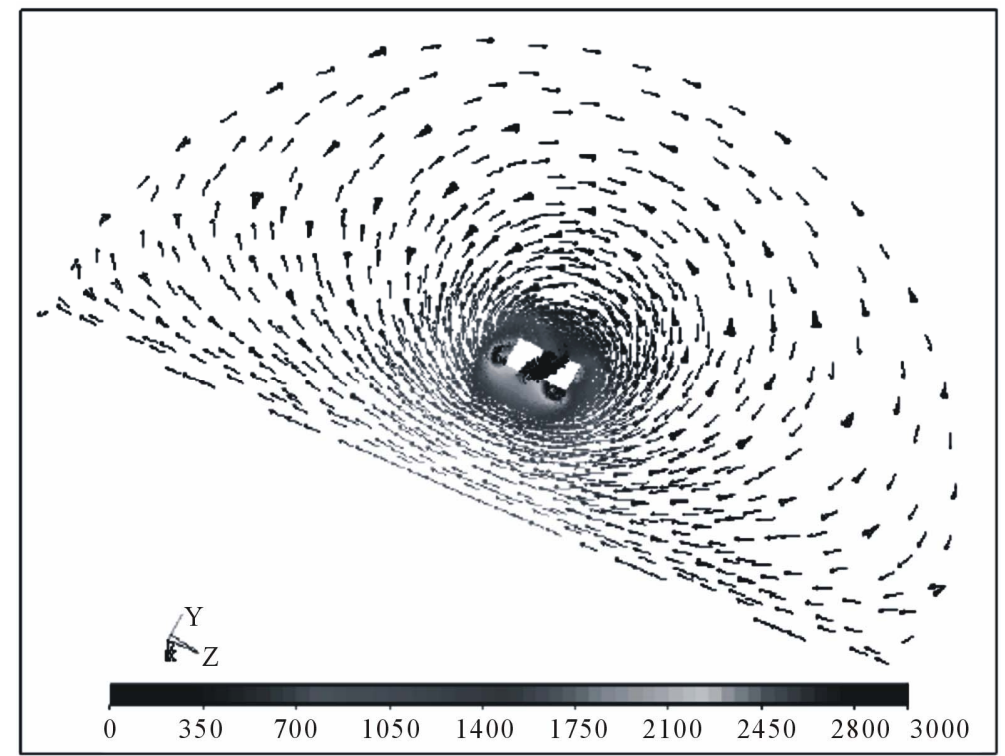

(c)

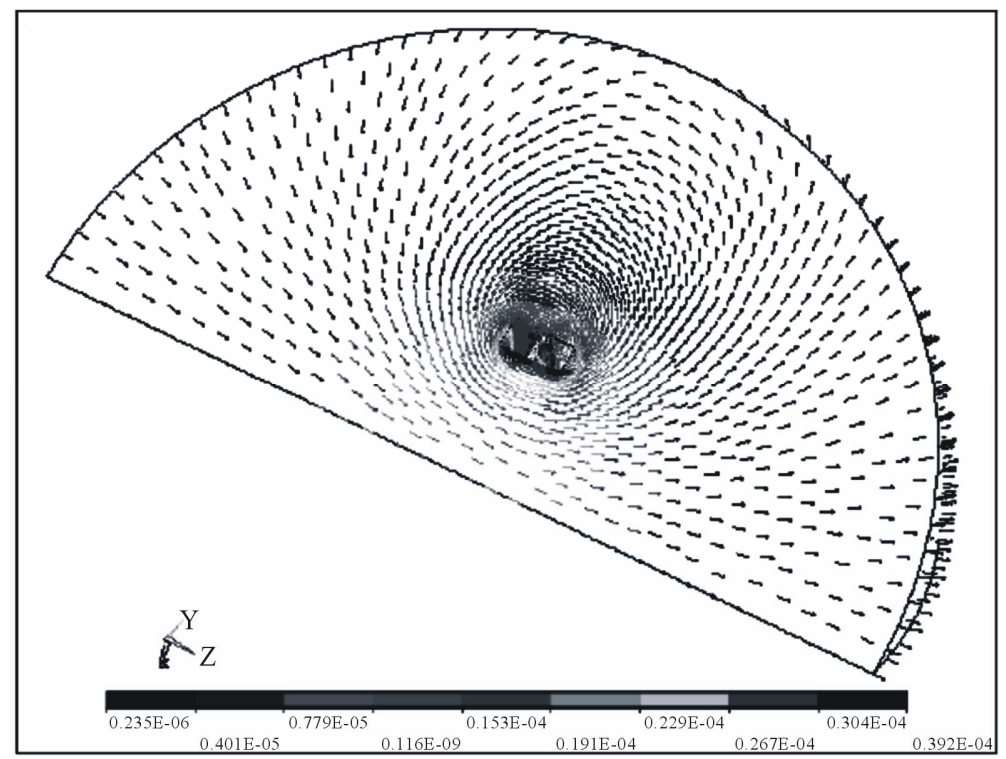

(d)

Figure 1. (a) Model of toroidal coils and segment of their close vicinity; (b) Section through model, with evaluation points marked out; (c) Distribution of E-field intensity (scale in $\mathrm{V} / \mathrm{m}$ ) at the instant its maximum is reached, i.e. the case of leading edge of excitation current pulse see; (d) Distribution of PVMP-field (scale in Wb/m) for toroidal coils without ferromagnetic cores (limiting condition of the calculation). 


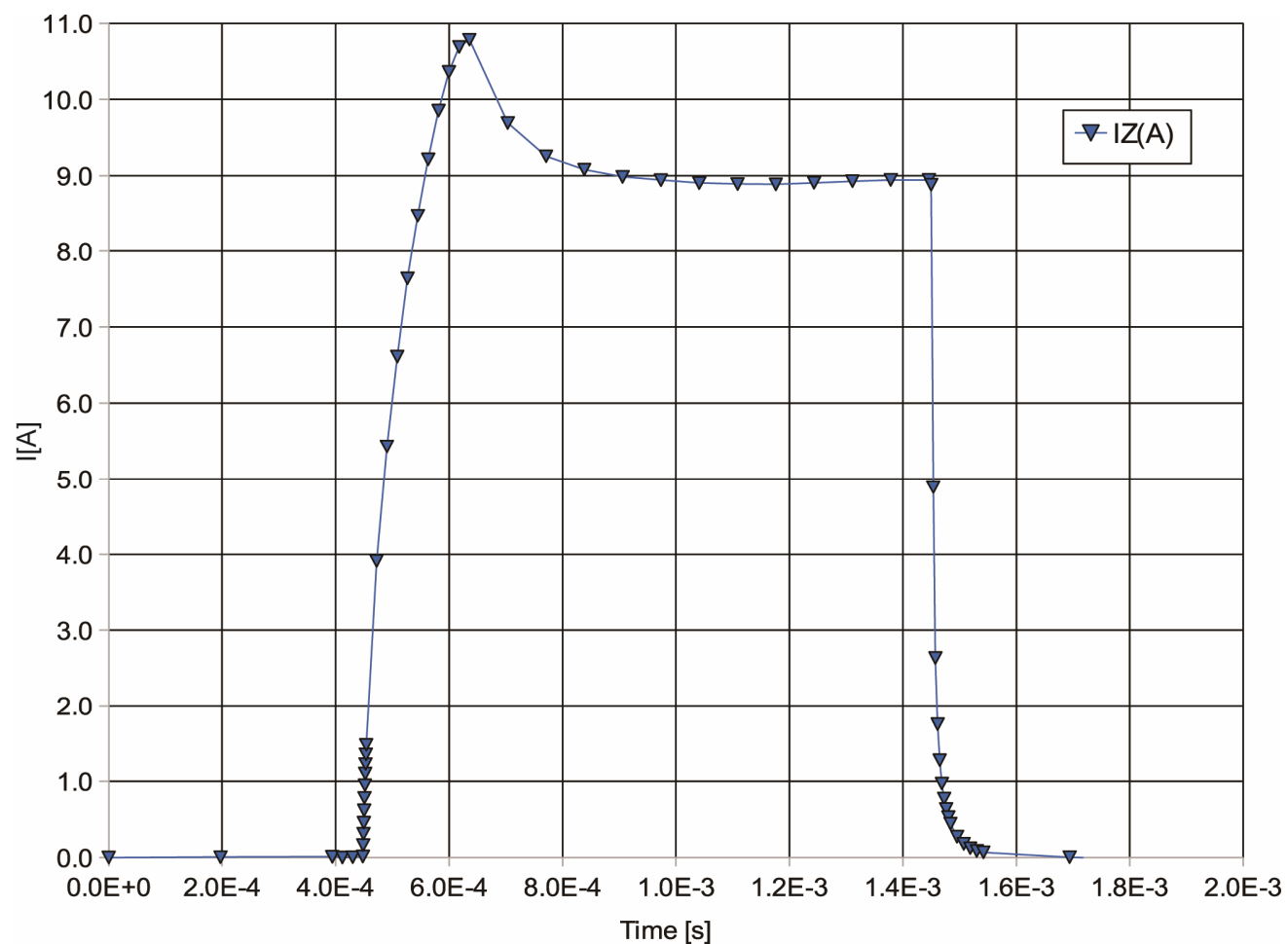

(a)

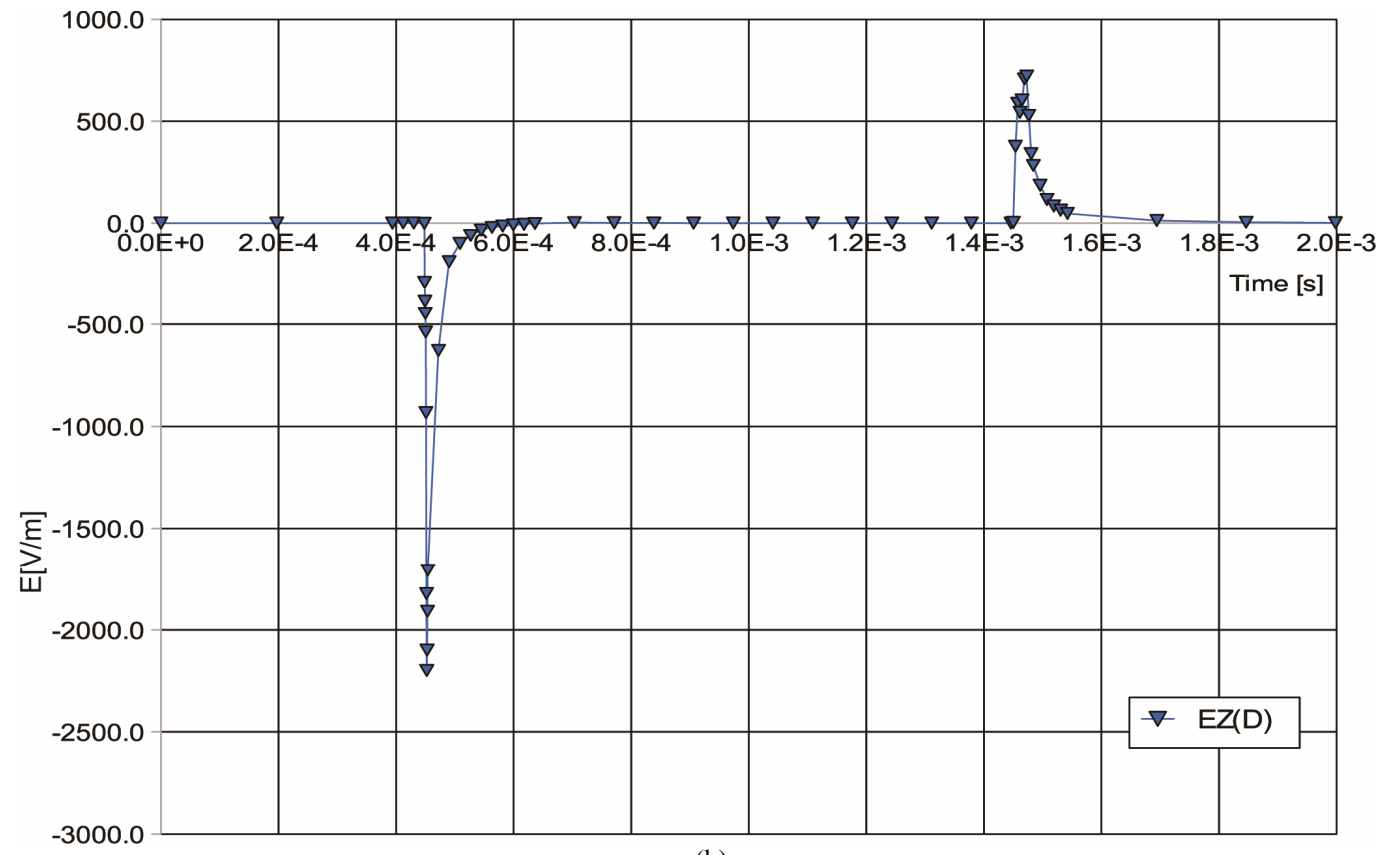

(b)

Figure 2. (a) Waveform of current pulse of width $T_{\text {pulse }}=1 \mathrm{~ms}$ flowing through coils at point A; (b) Time dependence of E-field intensity at spot $D$ (evaluation points are marked out in Figure 1(b)).

scribed was carried out under these conditions.

\subsubsection{Measurement of E-Field Intensity}

The EMS-100 device (Maschek, Germany) was used to measure the pulsed E-field in the inner space of the applicator. Comparing the values from Figure 1(c) with the values from Figure 3(b) indicates an obvious agreement between the simulation- and the measurement-established values of E-field intensity, which allows the assumption that the distribution of the hypothetic PVMPfield is likely to correspond to the distribution according to Figure 1(d). The same device confirmed the almost 


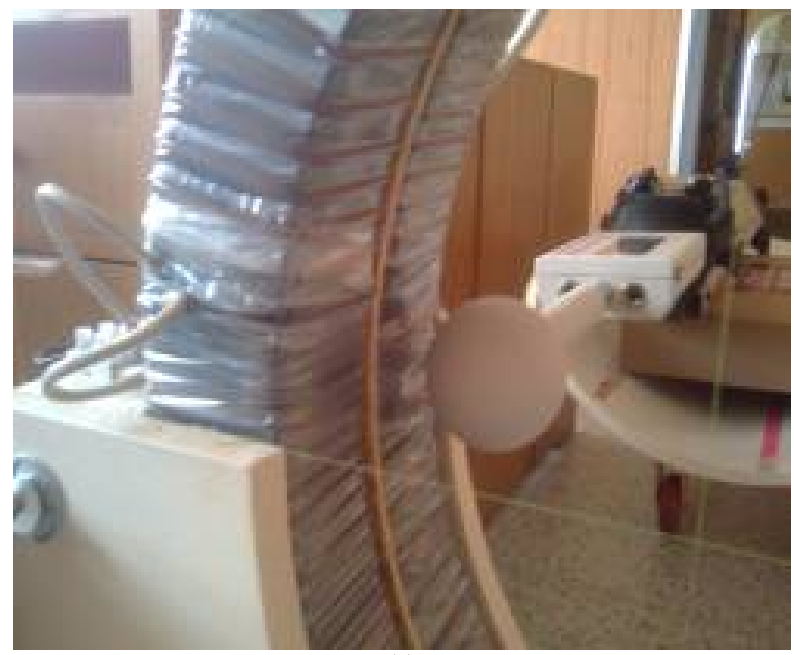

(a)

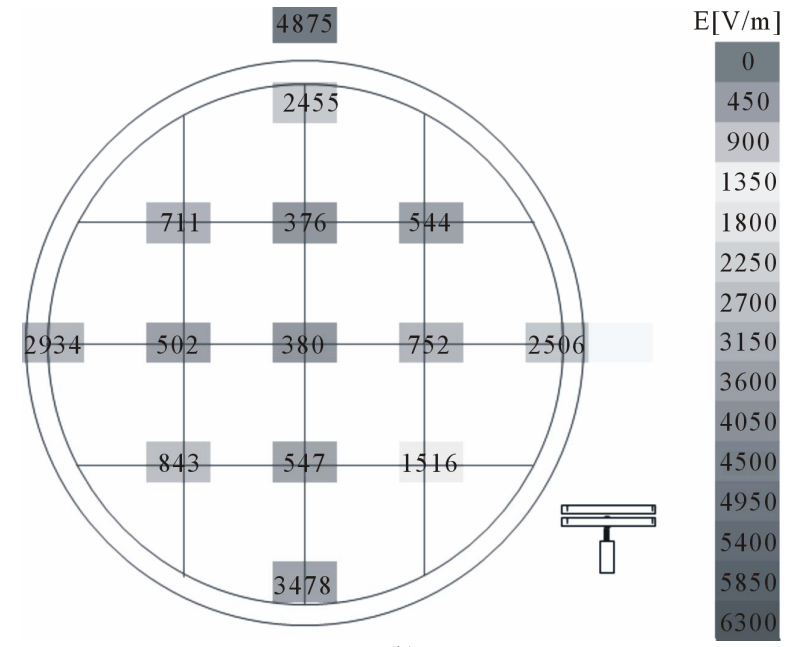

(b)

Figure 3. (a) Picture of a part of the device applicator with inserted measuring probe; (b) Distribution of E-field intensity in inner space of toroidal coils (scale in $\mathrm{V} / \mathrm{m}$ ).

zero outer pulsed B-field.

\subsection{General Description of the Biophysical Part of Experiment}

The essence of the biophysical part of the experiment consists in comparing the viability of living cells, in this experiment the Escherichia coli (E. coli) bacteria affected by PVMP-field with the viability of unaffected bacteria. Genetically modified E. coli K12 bacteria were used in the experiment. They are capable of bioluminescence, are resistant to ampicillin and their production of light is directly proportional to viability [3-5]. The bacterial suspension was divided between half the wells of a 96-wells microtitration plate (plate A) and half the wells of a 96-wells microtitration reference plate (plate B). Plate A was always placed in the device applicator as shown in Figure 4(a) while plate B was po- sitioned approximately $1 \mathrm{~m}$ outside the applicator action range.

\subsubsection{Preparation of the Experiment}

In the cultivation, deep-frozen bacterial culture $(\mathrm{t}=$ $-80^{\circ} \mathrm{C}$ ) was used, with $20 \mu \mathrm{l}$ of inoculum pipetted into Liquid Broth (LB) medium with ampicillin $(25 \mathrm{mg} / \mathrm{ml})$; subsequent cultivation proceeded for about $18 \mathrm{~h}$ under constant agitation at the temperature $t_{\text {susp }}=37^{\circ} \mathrm{C}$. For the purpose of cultivation, the suspension was diluted to an optical density of 0.5 Absorbance Unit (UA) at the light wavelength $\lambda=620 \mathrm{~nm}$. $200 \mu \mathrm{l}$ of this suspension were then pipetted into each of the 96 wells of the microtitration plate A. Pipetting was performed columnwise, using a multi-channel pipette. To rule out the possibility that pipetting might affect the results obtained (e.g. suspension sedimentation), the luminescence of all samples was measured for a period of three cycles (the time necessary for one cycle of measuring the bacteria luminescence in all the 96 wells is 132 seconds) using the LM01-T luminometer (Immunotech, Czech Republic). The measurement was carried out prior to the beginning of each test for the initial luminescence values of all wells to exhibit only minimum differences. Subsequently, half the 96 wells of plate A (4 rows - 48 wells) containing the bacterial suspension were taken out and put into plate $B$, which was placed outside the range of applicator action. Plate A with half the wells with bacterial suspension was placed in the inner space of the coils, as shown in Figure 4(a).

Due to the low thermal power loss of applicator coils and the considerable thermal capacity of their cores, the temperature of plate A was even under long-term functioning equal to the temperature of the vicinity of the position of plate $\mathrm{B}$, i.e. $\mathrm{t}_{\mathrm{vicin}}=(19-20)^{\circ} \mathrm{C}$ (any temperature effect can therefore be ruled out).

\subsubsection{Biophysical Experiment}

The effect of fields on the viability of bacteria $E$. coli measured in terms of bioluminescence was established
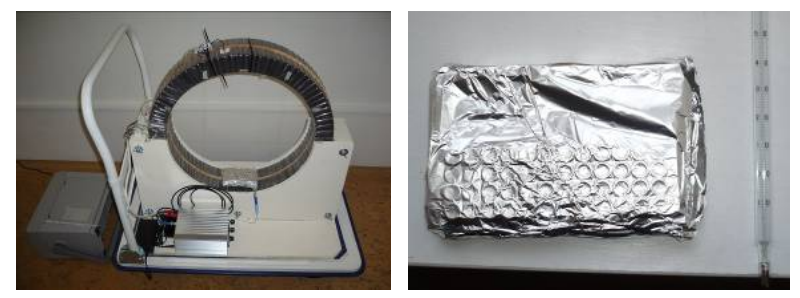

Figure 4. (a) Configuration of experimental device with applicator and pulse generator for the SHIELDED version of the experiment. Mounted on the bottom inner part of a pair of toroidal coils is the 96-wells titration plate $A$ in an earthed cover with 48 wells containing the suspension of bacteria $E$. coli; (b) Detail of plate B wrapped in a similar earthed cover with 48 wells filled with bacterial suspension. 
using a luminometer, which detects their luminescence in Relative Light Units (RLU). The dependence of RLU values on bacteria viability is linear. After a 4-hour application the wells of plate $B$ were placed back into plate A, after which the complete set of 96 wells was put into the luminometer, where continuous measurement proceeded for 2 hours.

The first values were obtained in a period of $2 \mathrm{~s}$ and then others with a 132 seconds measuring cycle. For the above setting of device parameters (Sub-Heading 2.1.2.) a total of 6 tests were performed. Each test always yielded 48 RLU values of irradiated and 48 RLU values of reference bacteria in $2 \mathrm{~s}$ after the measurement start.

To rule out potential effect of remanent magnetism of toroidal coil cores on the viability of bacteria placed in the measuring space of the applicator, a set of 6 measurements were carried out with the generator turned off.

\section{Statistical Data Evaluation}

The viability of bacteria was evaluated via the variance analysis with completely randomized blocks, separately for individual types of the test sets: NOT SHIELDED, SHIELDED and SWITCHED OFF. Data from these test sets were subdivided into blocks (always data measured in one day and from one bacterial suspension) with the $\mathrm{B} / \mathrm{A}$ factor (reference/measurement) regarded as fixed. The variance homogeneity was tested using the combined Cochran-Hartley-Bartlett test while the normality of residuals was assessed via the normal distribution graph. In case the respective F-test was significant $(\mathrm{P}<$ 0.05 ), the differences between mean values were evaluated using the post hoc Scheffé ${ }_{0.05}$ test. Within individual blocks the percentage difference between the application and the reference was calculated and these data were the subject of one-way variance analysis, where the fixed factor was the type of test set. The procedures of verifying the variance homogeneity and the normality of residuals were the same as described above; the differences between mean values were tested by means of two orthogonal contrasts: 1) SHIELDED vs. NOT SHIELDED, 2) mean values of the SHIELDED and NOT SHIELDED vs. the SWITCHED OFF. Statistically they are given in the form: mean $\pm \mathrm{SD}$ Standard Deviation, $\mathrm{n}=$ the number of samples-i.e. 6 test-repeats $\times 48$ RLU values $=288$, similarly $3 \times 48=144$ ). All the calculations were made using the STATISTICA 9.0 (StatSoft Inc., Tulsa, OK, USA) program.

\section{Results}

The experiments were divided into three types of test sets. Each application took 4 hours.

1) The set of type NOT SHIELDED tests (the two microtitration plates had their wells open). Altogether 6 test-repeats were carried out; each test yielded 48 RLU values for the application and 48 values for the reference, in 2 seconds after the beginning of measurement in the luminometer. In the NOT SHIELDED tests there is a statistically significant difference (the Scheffé ${ }_{0.05}$ test) between the viability of the bacteria in plate A exposed to applicator radiation $(153,244 \pm 65,280 ; n=288)$ and the reference bacteria in plate $\mathrm{B}(169,980 \pm 52,363 ; \mathrm{n}=$ 288). The viability of bacteria affected by the E-field and the PVMP-field reached $87.77 \%$ of the luminescence of reference bacteria.

2) The set of type SHIELDED tests (the two microtitration plates were wrapped in earthed Al foil for shielding against the E-field). A total of 6 repeats of each test yielded 48 RLU values for the application and 48 values for the reference, in 2 seconds after the measurement beginning. In the SHIELDED tests there is a statistically significant difference (the Scheffé ${ }_{0.05}$ test) between the viability of the bacteria in plate A exposed to applicator radiation $(75,652 \pm 32,059 ; \mathrm{n}=288)$ and the reference bacteria in plate $\mathrm{B}(99,726 \pm 34,605 ; \mathrm{n}=288)$. The viability of bacteria irradiated by the PVMP-field alone reached $76.71 \%$ of the luminescence of reference bacteria.

3) The set of type SWITCHED OFF tests. The plates with wells were positioned the same as in the preceding tests, with plate B outside the applicator range and plate A in the space of toroidal coils. Altogether three repeats were performed; each test yielded $48 \mathrm{RLU}$ values for the application and 48 values for the reference, in 2 seconds after the measurement beginning. In the case of the SWITCHED OFF tests there is no statistically significant difference (the Scheffe ${ }_{0.05}$ test) between the viability of the bacteria of plate A located in the applicator $(36,967 \pm$ $11,708 ; \mathrm{n}=144)$ and the reference bacteria in plate $\mathrm{B}$ $(36,689 \pm 8088 ; n=144)$. The viability of bacteria in plate A reached $100.63 \%$ of the luminescence of reference bacteria.

\section{Summary of Results}

In the case the device was switched off-type SWITCHED OFF test set - there was no applicator field to act on the bacteria in plate A, and difference between the bacterial culture of plate A and that of plate B was negligible. In the type NOT SHIELDED test set configuration the viability of the bacteria of plate A was, after 4 hours of irradiation, $\mathbf{8 7 . 7 7 \%}$ against the viability of the bacteria of plate $\mathrm{B}$. In the type SHIELDED test set configuration, when only the PVMP-field was acting, the viability of the irradiated bacteria of plate $A$ was only $\mathbf{7 6 . 7 1 \%}$ of the viability of the bacteria of plate B. The previous Figure 5 illustrates the results for the three sets of tests. 


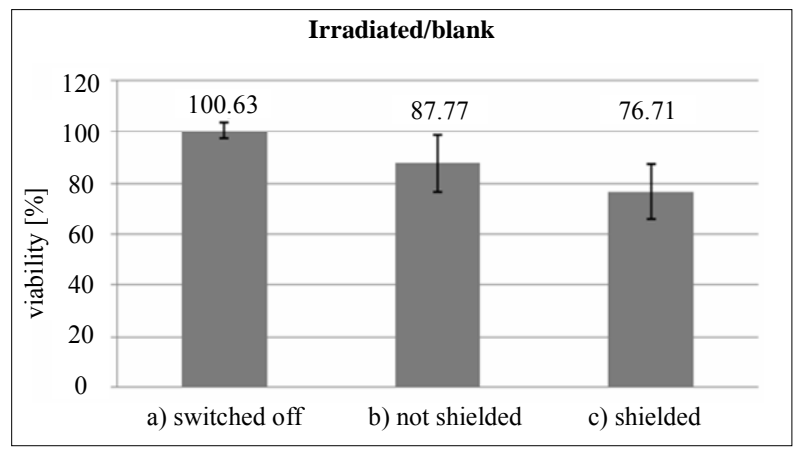

Figure 5. Graphical illustration of the effect of pulsed fields on the viability of bacteria $E$. coli for three different types of test sets, when: a) Experimental device was out of operation; b) Experimental device was in operation, with notshielded plate $A$; and c) With shielded plate $A$.

\section{Discussion and Conclusion}

Confirming the existence of the PVMP-field has only been possible by using a completely novel device with a relatively large applicator, acquiring data on the distribution of fields of force in the vicinity of pulse-supplied toroidal coils, employing computer simulations, and carrying out precise biophysical measurements in cooperation with the Institute of Experimental Biology, Department of Animal Physiology and Immunology, Faculty of Science, Masaryk University in Brno. Convincing results have been obtained, which allow claiming that the findings represent:

1) The discovery of statistically provable existence of the PVMP together with the manner and method of its indication;

2) The detection of quite an intensive action of the PVMP-field alone on bacteria $E$. coli, provided this field exists without the presence of an accompanying E-field.

An indispensable step in further research will be to establish the very reason for the effect of the PVMP-field on the viability of bacteria E. coli and, generally, on any living cells.

\section{REFERENCES}

[1] A. Shadowitz, "The Electromagnetic Field," Dover Publications Inc., New York, 1988.

[2] I. Rampl, V. Boudný, M. Číž, A. Lojek and P. Hyršl, "Pulse Vector Magnetic Potential and Its Influence on Live Cells," Proceedings of the International Conference on eHealth, Telemedicine, and Social Medicine, Cancun, 1-7 February 2009, pp. 99-107.

[3] P. Hyršl, S. Buchtíková, L. Vojtek, L. Palko and I. Rampl, "Bioluminescent Bacteria as Sensitive Tool for Induced Vector Magnetic Field and Induced Magnetic Field Measurements," XXXIII. Medicinal Biophysical Days, Mikulov, 2-4 June 2010, p. 28.

[4] P. Hyršl, M. Číž and A. Lojek, "Comparison of the Bioluminescence of Photorhabdus Species and Subspecies Type Strains," Folia Microbiologica, Vol. 49, No. 5, 2004, pp. 539-542. doi:10.1007/BF02931530

[5] L. Vojtek, P. Dobeš and P. Hyršl, "Antibacterial Methods Based on Bioluminescent Bacteria," Zoological Days, Brno, 17-18 February 2011, p. 249. 